JOURNAL OF THE

AMERICAN MATHEMATICAL SOCIETY

Volume 13, Number 1, Pages 129-148

S 0894-0347(99)00316-1

Article electronically published on September 13, 1999

\title{
COMBINATORIAL LAPLACIANS OF MATROID COMPLEXES
}

\author{
W. KOOK, V. REINER, AND D. STANTON
}

\section{INTRODUCTION}

For any finite simplicial complex $K$, one can define Laplace operators $\Delta_{i}$ which are combinatorial analogues of the Laplace operators on differential forms for a Riemannian manifold. The definition (as in $[6,7]$ ) is as follows. Let $C_{i}$ be the $\mathbb{R}$-vector space of (oriented) simplicial $i$-chains in $K$ with real coefficients, and $\partial_{i}: C_{i} \rightarrow C_{i-1}$ the usual simplicial boundary map. Endow $C_{i}$ with an inner product by declaring the oriented simplices to form an orthonormal basis of $C_{i}$, so that we may identify $C_{i}$ with its dual $C_{i}^{*}$. The adjoint to $\partial_{i}$ with respect to this inner product is the transpose $\partial_{i}^{T}$ or the coboundary map $\delta_{i-1}: C_{i-1} \rightarrow C_{i}$. Define $\Delta_{i}: C_{i} \rightarrow C_{i}$ by

$$
\Delta_{i}=\delta_{i-1} \partial_{i}+\partial_{i+1} \delta_{i}
$$

The main result about combinatorial Laplacians is an analogue of a fact from Hodge theory, namely that the kernel or 0-eigenspace of $\Delta_{i}$ is naturally isomorphic to the homology group $H_{i}(K ; \mathbb{R})[6$, Proposition 2.1]. The computational advantage of considering $\Delta_{i}$ is that one can compute numerical approximations to the spectrum of $\Delta_{i}$ and find its 0-eigenspace relatively quickly, using well-established fast methods for spectra of real symmetric matrices [6,7].

These methods are particularly effective if one knows that the spectra of the $\Delta_{i}$ are integral, which is not often the case. However, the spectra are integral for the well-studied chessboard complexes, as shown in [8]. There the authors used the symmetry groups acting on the complexes for a clever representation-theoretic calculation, which gives the spectrum and in particular its integrality.

The main result of this paper (Corollary 18) proves that the spectra of all the Laplacians are again integral when $K$ is a matroid complex, that is, when $K$ is the complex of independent sets in a matroid. The result follows from a surprising orthogonal decomposition of the chain groups $C_{i}$ (Theorem 16) which is in general finer than the eigenspace decomposition for the Laplacians. We remark that even though the combinatorial Laplacians have usually been defined only over fields of characteristic zero, all of our results are still true over the integers.

One corollary (Corollary 10) to our results is that for a matroid complex, only a very small part of the information in the spectra of the Laplacians (namely the

Received by the editors July 16, 1997 and, in revised form, June 30, 1999.

2000 Mathematics Subject Classification. Primary 05B35.

Key words and phrases. Matroid, matroid complex, Laplacian, internal activity, external activity.

The second author was supported by Sloan Foundation and University of Minnesota McKnight Land Grant Fellowships. The third author was supported by NSF grant DMS-9400510.

(C)1999 American Mathematical Society 
multiplicity of the largest eigenvalue in each $\Delta_{i}$ ) is sufficient to recover the characteristic polynomial of the matroid. This adds to the already large list (see e.g. [4]) of interpretations of the characteristic polynomial of a matroid, and can be whimsically rephrased by saying that one can "hear" the characteristic polynomial of a matroid.

The paper is structured as follows. Section 2 deals with some combinatorial preliminaries that do not involve Laplacians, but are really the combinatorial manifestations that foreshadow Theorem 16. Section 3 introduces the combinatorial Laplacians and proves some easy basic facts about their behavior for matroid complexes. Section 4 contains the main results, which require a slightly deeper analysis, aided by the technique of exterior face rings for simplicial complexes [2]. Section 5 discusses some well-known examples of matroids having large symmetry groups.

\section{Combinatorial PReliminaries}

We refer the reader to the excellent article [1] for almost all of the definitions and notions from matroid theory and matroid complexes that we will use.

Let $M$ be a loopless matroid with a chosen linear ordering $\omega$ on its ground set $E$. In the usual way there is associated to $M$ a rank function $r(A)$ and a closure operator $A \mapsto \bar{A}$ on subsets $A \subseteq E$, and the set of closed subsets or flats forms a geometric lattice denoted $L(M)$.

Recall that for a base $B$ of $M$, an element $b$ in $B$ of $M$ gives rise to a unique basic bond bo $o_{M}(B, b)$, and an element $e$ in $E-B$ gives rise to a unique basic circuit $c i_{M}(B, e)$. We say that $b$ is internally active in $B$ if it is the $\omega$-smallest element of $b o_{M}(B, b)$, and $e$ is externally active if it is the $\omega$-smallest element of $c i_{M}(B, e)$. The internal activity $i(B)$ of the base $B$ is the number of internally active elements in $B$, and its external activity $e(B)$ is defined similarly.

The following combinatorial result was discovered independently by Etienne and Las Vergnas [5]. It will be used in a later proof (Theorem 16) for a dimensioncounting argument.

Theorem 1. In any ordered matroid $(M, \omega)$, every base $B$ has a unique disjoint decomposition $B=B_{1} \sqcup B_{2}$ with the following properties:

- $B_{1}$ is a base of internal activity 0 for the flat $V:=\overline{B_{1}}$ which it spans, and

- $B_{2}$ is a base of external activity 0 for the contracted matroid $M / V$.

Proof. The existence of such a decomposition is provided by the following algorithm, which takes the base $B$ as input, and produces such a pair $\left(B_{1}, B_{2}\right)$.

Algorithm. Step 1. Set $B_{1}=B, B_{2}=\varnothing$.

Step 2. Let $V=\overline{B_{1}}$.

Step 3. Find an internally active element $b$ for $B_{1}$ as a base of the flat $V$.

If no such element $b$ exists, then stop and output the pair $\left(B_{1}, B_{2}\right)$.

If such a $b$ exists, then set $B_{1}:=B_{1}-\{b\}, B_{2}:=B_{2} \cup\{b\}$, and return to Step 2 .

It is clear that the algorithm terminates, since each time one returns to Step 2, the cardinality of $B_{1}$ has decreased. To see that the output is a decomposition as in the theorem, note that $B_{1}$ will be a base for $V$ of internal activity 0 by construction, so the first property is clearly satisfied.

Therefore it remains to show the second property, i.e. that $B_{2}$ is a base of external activity 0 for $M / V$. For this we will show that given any $e \in E-V-B_{2}$ 
the circuit $c i_{M / V}\left(B_{2}, e\right)$ contains an $\omega$-smaller element than $e$. Since $c i_{M / V}\left(B_{2}, e\right)$ is a circuit in $M / V$, the set $c i_{M / V}\left(B_{2}, e\right) \cup B_{1}$ is dependent in $M$, and therefore contains some circuit of $M$ of the form $c i_{M / V}\left(B_{2}, e\right) \cup B_{1}^{\prime}$ where $B_{1}^{\prime} \subseteq B_{1}$. Denote by $\left(B_{1}^{(i)}, B_{2}^{(i)}\right)$ the sets $\left(B_{1}, B_{2}\right)$ at the $i^{\text {th }}$ stage of the above algorithm, so $B_{1}^{(0)}=$ $B, B_{2}^{(0)}=\varnothing$. Let $i$ be such that $c i_{M / V}\left(B_{2}, e\right)-\{e\} \subseteq B_{1}^{(i)}$ but $c i_{M / V}\left(B_{2}, e\right)-\{e\} \nsubseteq$ $B_{1}^{(i+1)}$. This means that some element $b_{2}$ of $c i_{M / V}\left(B_{2}, e\right)-\{e\}$ is thrown out of $B_{1}^{(i)}$ and into $B_{2}^{(i+1)}$ at this stage because $b_{2}$ is internally active for $B_{1}^{(i)}$. Note that $c i_{M / V}\left(B_{2}, e\right)-\{e\} \cup B_{1}^{\prime} \subseteq B_{1}^{(i)}$ and consequently $b_{2} \in c i_{V}\left(B_{1}^{(i)}, e\right)$ with $b_{2}$ being $\omega$-smaller than $e$.

To show uniqueness of the decomposition, we define a map $f$ between two sets:

- the set of triples $\left(V, B_{1}, B_{2}\right)$ where $V$ is a flat of $M, B_{1}$ is a base of internal activity 0 for $V$, and $B_{2}$ is a base of external activity 0 for $M / V$, and

- the set of bases $B$ of $M$.

The map $f$ sends the triple $\left(V, B_{1}, B_{2}\right)$ to the base $B:=B_{1} \sqcup B_{2}$. Uniqueness of the decomposition is equivalent to injectivity of $f$, and since the above algorithm shows that $f$ is surjective, it suffices to show that the two sets have the same cardinality, i.e. that

$$
\mid \text { bases of } M\left|=\sum_{\text {flats } V \text { of } M}\right| \begin{gathered}
\text { bases } B_{1} \text { of } V \\
\text { with internal activity } 0
\end{gathered}|\times| \begin{gathered}
\text { bases } B_{2} \text { of } M / V \\
\text { with external activity } 0
\end{gathered} \mid \text {. }
$$

To see this, recall from [1, Theorem 7.8.4] that the number of bases of a matroid $M$ having internal activity 0 is, up to sign, the (reduced) Euler characteristic $\tilde{\chi}(I N(M))$ of the independence complex $I N(M)$, and hence is equal to the alternating sum $\sum_{I}(-1)^{r(M)-|I|}$ where $I$ ranges over the independent sets of $M$. Recall also from [1, Proposition 7.4.7] that the number of bases of a matroid $M$ having external activity 0 is the absolute value of the Möbius function $\left|\mu_{L(M)}(\hat{0}, \hat{1})\right|$ for the geometric lattice $L(M)$. Therefore starting with the right-hand side in equation (2.1), we can manipulate as follows:

$$
\begin{aligned}
& \sum_{\text {flats } V \text { of } M}\left|\begin{array}{c}
\text { bases } B_{1} \text { of } V \\
\text { with internal activity } 0
\end{array}\right| \times\left|\begin{array}{c}
\text { bases } B_{2} \text { of } M / V \\
\text { with external activity } 0
\end{array}\right| \\
& =\sum_{\text {flats } V \text { of } M}\left(\sum_{\text {independent sets } I \subseteq V}(-1)^{r(V)-|I|}\right)\left|\mu_{L(M / V)}(\hat{0}, \hat{1})\right| \\
& =\sum_{\text {independent sets } I \subseteq E}(-1)^{r(M)-|I|} \sum_{\bar{I} \leq V \leq \hat{1}}(-1)^{r(M)-r(V)}\left|\mu_{L(M)}(V, \hat{1})\right| \\
& =\sum_{\text {independent sets } I \subseteq E}(-1)^{r(M)-|I|} \sum_{\bar{I} \leq V \leq \hat{1}} \mu_{L(M)}(V, \hat{1}) \\
& =\sum_{\text {independent sets } I \text { with } \bar{I}=M}(-1)^{r(M)-|I|} \\
& =\sum_{\text {bases } B \text { of } M}(-1)^{r(M)-|B|} \\
& =\mid \text { bases of } M \mid \text {. }
\end{aligned}
$$


Here we have used various facts, such as the defining recurrence for the Möbius function, the fact that the Möbius function of the geometric lattice $L(M)$ alternates in sign, and the fact that the interval $[V, \hat{1} \mid$ in $L(M)$ is isomorphic to the lattice of flats $L(M / V)$ for the contracted matroid $M / V$.

Based on Theorem 1, we define the spectrum polynomial of $M$

$$
\operatorname{Spec}_{M}(t, q):=\sum_{\text {independent sets } I} t^{r(\bar{I})} q^{\mid \overline{\left|I_{1}\right|}}
$$

where $I_{1}$ denotes the output of the decomposition algorithm $I \mapsto\left(I_{1}, I_{2}\right)$ when $I$ is considered as a base for the flat $\bar{I}$ it spans, and the ordering on the ground set in $\bar{I}$ is induced from the original ordering $\omega$ on $E$.

The reason for the terminology "spectrum polynomial" is that $\operatorname{Spec}_{M}(t, q)$ carries the same information as the spectra of the Laplacians for the matroid complex of $M$ (Corollary 18). The simpler fact that $\operatorname{Spec}_{M}(t, q)$ does not depend on the chosen ordering $\omega$ of the ground set is easy to see from the following alternate expression:

$$
\begin{aligned}
\operatorname{Spec}_{M}(t, q) & =\sum_{\text {flats } V} t^{r(V)} \sum_{\text {independent sets } I \text { spanning } V} q^{\left|\overline{I_{1}}\right|} \\
& =\sum_{\text {flats } V} t^{r(V)} \sum_{\text {flats } V^{\prime} \subseteq V}\left|\tilde{\chi}\left(I N\left(V^{\prime}\right)\right)\right| \cdot\left|\mu_{L(M)}\left(V^{\prime}, V\right)\right| q^{\left|V^{\prime}\right|} .
\end{aligned}
$$

The last equality follows from the same facts used to show that the map $f$ was a bijection in the proof of Theorem 1.

A straightforward consequence of the definition is that for a direct sum $M_{1} \oplus M_{2}$ of two matroids $M_{1}, M_{2}$, we have

$$
\operatorname{Spec}_{M_{1} \oplus M_{2}}(t, q)=\operatorname{Spec}_{M_{1}}(t, q) \cdot \operatorname{Spec}_{M_{2}}(t, q),
$$

a property reminiscent of the Tutte polynomial of a matroid. Recall that the Tutte polynomial $T_{M}(x, y)$ may be defined by

$$
T_{M}(x, y):=\sum_{\text {bases } B \text { of } M} x^{i(B)} y^{e(B)}
$$

where $i(B), e(B)$ are the internal and external activity of the base $B$. The Tutte polynomial plays an important role in matroid theory; see [4]. Since the Tutte polynomial and spectrum polynomial are both related to the notions of internal and external activity, it is tempting to compare them.

Question 2. How does the spectrum polynomial compare with the Tutte polynomial in distinguishing non-isomorphic matroids? Can two matroids have the same spectrum polynomial and different Tutte polynomials?

We know that there are non-isomorphic matroids that have the same spectrum polynomial. For example, the matroids $M_{1}, M_{2}$ represented in characteristic zero by the affine point configurations shown in Figure 1 have the same spectrum polynomial

$$
1+6 t+t^{2}\left(13+2 q^{3}\right)+t^{3}\left(8+2 q^{3}+8 q^{6}\right)
$$

and the same Tutte polynomial [4, Problem 6.1(b)]

$$
x^{3}+y^{3}+3 x^{2}+3 y^{2}+2 x y+4 x+4 y .
$$




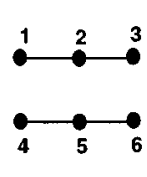

$\mathbf{M}_{1}$

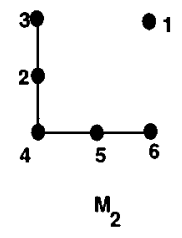

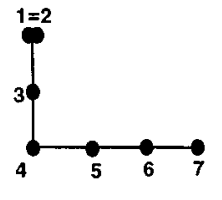

$M_{4}$

FiguRE 1. Matroids $M_{1}$ and $M_{2}$ have the same Tutte polynomial and the same spectrum polynomial. Matroids $M_{3}$ and $M_{4}$ have the same Tutte polynomial but different spectrum polynomials.

On the other hand, there are non-isomorphic matroids that have the same Tutte polynomial, but not the same spectrum polynomial. For example, the matroids $M_{3}, M_{4}$ shown in Figure 1 have the same Tutte polynomial [4, Example 6.2.18]

$$
y^{4}+3 y^{3}+2 x y^{2}+x^{2} y+x^{3}+4 y^{2}+5 x y+3 x^{2}+2 x+2 y,
$$

but different spectrum polynomials

$$
\begin{aligned}
\operatorname{Spec}_{M_{3}}(t, q)= & 1+t\left(6+q^{2}\right)+t^{2}\left(11+3 q^{2}+2 q^{3}+4 q^{4}\right) \\
& +t^{3}\left(6+2 q^{2}+2 q^{3}+4 q^{4}+10 q^{7}\right), \\
\operatorname{Spec}_{M_{4}}(t, q)= & 1+t\left(6+q^{2}\right)+t^{2}\left(11+4 q^{2}+5 q^{4}\right)+t^{3}\left(6+3 q^{2}+5 q^{4}+10 q^{7}\right) .
\end{aligned}
$$

We see no reason to expect that the spectrum polynomial determines the Tutte polynomial, but we have no counterexamples.

Since the Tutte polynomial is easy to compute by the deletion-contraction recurrence

$$
T_{M}(x, y)=T_{M-e}(x, y)+T_{M / e}(x, y),
$$

the following question is natural:

Question 3. Is there some way to use deletion-contraction to compute the spectrum polynomial $\operatorname{Spec}_{M}(t, q)$ ?

The examples $M_{3}, M_{4}$ above show that there can be no formula for $\operatorname{Spec}_{M}(t, q)$ purely as a function of $\operatorname{Spec}_{M-e}(t, q), \operatorname{Spec}_{M / e}(t, q)$, since $M_{3}-e \cong M_{4}-e$ and $M_{3} / e \cong M_{4} / e$ for the element $e$ of the ground set labelled 7 in each figure. Nevertheless, there might exist some more complicated procedure for computing the spectrum polynomial of $M$ from those of a deletion $M-e$ and contraction $M / e$, which somehow uses the way in which $M-e$ and $M / e$ "glue together" to produce $M$.

\section{LAPLACIANS}

The goal of this section is to define the combinatorial Laplacians of matroid complexes, and prove some easy facts about them.

Let $I N(M)$ denote the independence complex of the matroid $M$, i.e. the simplicial complex on vertex set $E$ whose simplices are the independent sets of $M$. Let $C_{i}^{M}$ denote the $i^{\text {th }}$ (oriented) simplicial chain group of $I N(M)$ with $\mathbb{R}$ coefficients, $\partial_{i}^{M}: C_{i}^{M} \rightarrow C_{i-1}^{M}$ the usual simplicial boundary map, and $\tilde{H}_{i}^{M}$ the $i^{\text {th }}$ (reduced) homology group with $\mathbb{R}$ coefficients. By choosing the standard inner product $\langle\cdot, \cdot \cdot\rangle$ on $C_{i}^{M}$ in which the oriented simplices are an orthonormal basis, we can identify $C_{i}^{M}$ with its dual space of cochains. Then the simplicial coboundary map $\delta_{i}^{M}$ 
which is the adjoint of $\partial_{i}^{M}$ may be thought of as a map $C_{i-1}^{M} \rightarrow C_{i}^{M}$. Define the $i^{t h}$ combinatorial Laplacian to be the operator $\Delta_{i}^{M}: C_{i}^{M} \rightarrow C_{i}^{M}$ given by

$$
\Delta_{i}^{M}:=\delta_{i-1}^{M} \partial_{i}^{M}+\partial_{i+1}^{M} \delta_{i}^{M} .
$$

It is not hard to show [6] that the kernel or 0-eigenspace $\operatorname{ker}\left(\Delta_{i}^{M}\right)$ is isomorphic to the homology $\tilde{H}_{i}^{M}$. In our case, there is not just an isomorphism but an equality $\operatorname{ker}\left(\Delta_{r(M)-1}^{M}\right)=\tilde{H}_{r(M)-1}^{M}$ since there are no $(r(M)-1)$-dimensional boundaries. Furthermore, since $I N(M)$ is shellable [1, Theorem 7.3.3] and hence has only $(r(M)-1)$-dimensional homology, we have that $\operatorname{ker}\left(\Delta_{i}^{M}\right)=0$ for $i<r(M)-1$, a fact we will recover for different reasons shortly (Corollary 7 ).

It is easy to compute the following explicit description for $\Delta_{i}^{M}$. For each independent $(i+1)$-set $I$ of $M$, if we let $[I]$ denote the oriented simplex which places the elements of $I$ in $\omega$-order, then

$$
\Delta_{i}^{M}[I]=(|I|+|E-\bar{I}|) \cdot[I]+\sum_{\substack{I^{\prime}=I-\{e\}+\left\{e^{\prime}\right\} \\ I^{\prime} \cup I \text { dependent }}}(-1)^{d\left(I, I^{\prime}\right)}\left[I^{\prime}\right]
$$

where $d\left(I, I^{\prime}\right)$ is the number of elements in $I \cup I^{\prime}$ which lie strictly between $e, e^{\prime}$ under the $\omega$-ordering.

Remark 4. Apparently $\Delta_{i}^{M}$ depends on $\omega$. However, one can check that changing $\omega$ only conjugates $\Delta_{i}^{M}$ by a signed permutation matrix.

First a few simple observations.

Proposition 5. The chain group $C_{i}^{M}$ and the operator $\Delta_{i}^{M}$ decompose as a direct sum

$$
\begin{aligned}
C_{i}^{M} & =\bigoplus_{\text {rank }(i+1) \text { flats } V} C_{i}^{V}, \\
\Delta_{i}^{M} & =\bigoplus_{\text {rank }(i+1) \text { flats } V}\left(\Delta_{i}^{V}+|E-V| \cdot I_{V}\right) .
\end{aligned}
$$

Here $C_{i}^{V}$ is the subspace of $i$-chains supported on independent sets $I$ which are a base for the flat $V$, and $I_{V}$ is the identity map on this subspace, while $\Delta_{i}^{V}$ is the $i^{\text {th }}$ Laplacian for the restricted matroid $\left.M\right|_{V}$.

Proof. Looking at the explicit formula (3.1) for $\Delta_{i}^{M}$, one sees that the $\left(I, I^{\prime}\right)$ offdiagonal entry is non-zero only when $I$ and $I^{\prime}$ are bases of the same flat $V$. Furthermore, in this case the scalars $|E-\bar{I}|,\left|E-\overline{I^{\prime}}\right|$ are both equal to $|E-V|$.

As a consequence of the previous proposition, we really need only understand the top Laplacian $\Delta_{r(M)-1}^{M}$ acting on $C_{r(M)-1}^{M}$.

To this end, recall that the map $B \mapsto E-B$ gives a bijection between the bases for $M$ and the bases for its dual matroid $M^{*}$. Extend this bijection to a linear isomorphism

$$
P: C_{r(M)-1}^{M} \rightarrow C_{r\left(M^{*}\right)-1}^{M^{*}}
$$

by sending the oriented simplex $[B]$ to $(-1)^{\operatorname{ranksum}(B)}[E-B]$ where $\operatorname{ranksum}(B)$ is the sum of the ranks of the elements of $B$ in the $\omega$-order. 
Proposition 6.

$$
\Delta_{r(M)-1}^{M}+P^{-1} \Delta_{r\left(M^{*}\right)-1}^{M^{*}} P=|E| \cdot I_{M}
$$

where $I_{M}$ is the identity map on $C_{r(M)-1}^{M}$.

Proof. Examining the explicit formula (3.1) for $\Delta_{i}^{M}$ in the case $i=r(M)-1$, we see that the diagonal entries for $\Delta_{r(M)-1}^{M}, P^{-1} \Delta_{r\left(M^{*}\right)-1}^{M^{*}} P$ are $r(M),|E|-r(M)$, respectively. Consequently the assertion for the diagonal entries in the proposition is clear. For the off-diagonal entries, one needs to check that for any bases $B, B^{\prime}$ of $M$ satisfying $B^{\prime}=B-\{e\} \cup\left\{e^{\prime}\right\}$ we have

$$
(-1)^{\text {ranksum }(B)}(-1)^{\text {ranksum }\left(B^{\prime}\right)}(-1)^{d\left(B, B^{\prime}\right)}+(-1)^{d\left(E-B, E-B^{\prime}\right)}=0 .
$$

This verification is straightforward.

Corollary 7. For every $i$, the spectrum of Laplacian $\Delta_{i}$ is contained in the interval $[0,|E|]$ of $\mathbb{R}$, and will not contain 0 unless $i=r(M)-1$.

Proof. We know that $\Delta_{i}^{M}$ is non-negative definite since it is a sum of the two operators $\delta_{i-1}^{M} \partial_{i}^{M}$ and $\partial_{i+1}^{M} \delta_{i}^{M}$, which are both non-negative definite since they are of the form $A^{*} A$ (see [6, Proposition 2.1]). Therefore the spectrum of $\Delta_{i}^{M}$ is non-negative.

If we look at the special case of $i=r(M)-1$, then the previous proposition says that the spectrum of $\Delta_{r(M)-1}^{M}$ is obtained from the (non-negative) spectrum of $\Delta_{r\left(M^{*}\right)-1}^{M^{*}}$ by subtraction from $|E|$. Therefore the assertion of the corollary holds for $i=r(M)-1$. But then a glance at Proposition 5 shows that it also holds for the remaining $i$.

Proposition 6 shows that the spectrum of the top Laplacian $\Delta_{r(M)-1}^{M}$ completely determines the spectrum of the dual $\Delta_{r\left(M^{*}\right)-1}^{M^{*}}$. This suggests the following question:

Question 8. Is the same true for the lower-dimensional Laplacians $\Delta_{i}^{M}$, or do there exist two matroids $M_{1}, M_{2}$ for which $\Delta_{i}^{M_{1}}$ and $\Delta_{i}^{M_{2}}$ have the same spectra for all $i$, but some $\Delta_{j}^{M_{1}^{*}}$ and $\Delta_{j}^{M_{2}^{*}}$ have different spectra?

This also suggests a stronger question to which we also do not know the answer:

Question 9. To what extent does the top spectrum determine the lower spectra?

For a real number $\lambda$, let $\left(\Delta_{i}^{M}\right)_{\lambda}$ denote the $\lambda$-eigenspace for the operator $\Delta_{i}^{M}$. Corollary 7 shows that the largest possible value of $\lambda$ is the cardinality of the ground set $|E|$. Our next result gives an interesting interpretation for the multiplicities of this largest eigenvalue in the various Laplacians $\Delta_{i}^{M}$.

Recall that the characteristic polynomial of the matroid is defined by

$$
\chi_{M}(t):=\sum_{V \in L(M)} \mu_{L(M)}(\hat{0}, V) t^{r(M)-r(V)}
$$

where $\mu_{L(M)}$ is the Möbius function for the geometric lattice $L(M)$. The next corollary has the whimsical interpretation that you can "hear" the characteristic polynomial of $M$, if by analogy with vibrating drums, we imagine that the spectra of the various Laplacians $\Delta_{i}^{M}$ are heard in the frequencies emitted when one "bangs" a matroid complex! 


\section{Corollary 10.}

$$
\chi_{M}(-t)=(-1)^{r(M)} t^{r(M)-1} \sum_{i=-1}^{r(M)-1} \operatorname{dim}_{\mathbb{R}}\left(\Delta_{i}^{M}\right)_{|E|} t^{-i} .
$$

Proof.

$$
\begin{aligned}
& (-1)^{r(M)}(-t)^{r(M)-1} \sum_{i=-1}^{r(M)-1} \operatorname{dim}_{\mathbb{R}}\left(\Delta_{i}^{M}\right)_{|E|}(-t)^{-i} \\
& =(-1)^{r(M)}(-t)^{r(M)-1} \sum_{i=-1}^{r(M)-1} \sum_{\text {flats } V: r(V)=i+1} \operatorname{dim}_{\mathbb{R}}\left(\Delta_{i}^{V}\right)_{|V|}(-t)^{-i} \\
& =(-1)^{r(M)} \sum_{\text {flats } V} \operatorname{dim}_{\mathbb{R}}\left(\Delta_{r(V)-1}^{V}\right)_{|V|}(-t)^{r(M)-r(V)} \\
& =(-1)^{r(M)} \sum_{\operatorname{lftats} \operatorname{dim}_{\mathbb{R}}\left(\Delta_{r\left(V^{*}\right)-1}^{V^{*}}\right)_{0}(-t)^{r(M)-r(V)}} \sum_{\text {flats } V} \operatorname{dim}_{\mathbb{R}} \tilde{H}_{r\left(V^{*}\right)-1}\left(I N\left(V^{*}\right)\right)(-t)^{r(M)-r(V)} \\
& =(-1)^{r(M)} \sum_{\text {flats } V}\left|\mu_{L(V)}(\hat{0}, \hat{1})\right|(-t)^{r(M)-r(V)} \\
& =\sum_{V \in L(M)} \mu_{L(M)}(\hat{0}, V) t^{r(M)-r(V)} .
\end{aligned}
$$

The first equality uses Proposition 5. The second equality is just interchanging the order of summation. The third equality uses Proposition 6. The fourth equality uses the fact that the 0-eigenspace for the top Laplacian is the same as the top homology of the independence complex. The fifth equality uses the fact from $[1$, Theorem 7.8.1] that this top homology has dimension equal to the Möbius number for the geometric lattice of the dual matroid. The sixth equality uses the fact that the Möbius function for the geometric lattice $L(M)$ alternates in sign.

It is well-known that the Tutte polynomial $T_{M}(x, y)$ also specializes to the characteristic polynomial:

$$
\chi_{M}(t)=(-1)^{r(M)} T_{M}(1-t, 0) .
$$

The generating function

$$
\sum_{\text {independent sets } I \text { in } M} t^{|I|}=t^{r(M)} T_{M}\left(1+t^{-1}, 1\right)
$$

is another specialization of the Tutte polynomial which is clearly recoverable from the spectra of the various $\Delta_{i}^{M}$. This prompts the following question:

Question 11. How much can one "hear" of the Tutte polynomial of a matroid, i.e. how much of $T_{M}(x, y)$ is recoverable from knowledge of the spectra of the various Laplacians $\Delta_{i}^{M}$ ?

This question will turn out to be equivalent to Question 2 via Corollary 18. 


\section{Orthogonal decomposition of $C_{r(M)-1}^{M}$ AND the EXterior FACE Ring}

The goal of this section is to probe deeper into the spectra of the Laplacians $\Delta_{i}^{M}$, which by Proposition 5 reduces to understanding $\Delta_{r(M)-1}^{M}$. We will show that all of its eigenvalues are integers, and explain how the eigenspace decomposition of $C_{r(M)-1}^{M}$ with respect to $\Delta_{r(M)-1}^{M}$ is refined by a more fundamental orthogonal decomposition (Theorem 16) whose combinatorial manifestation is Theorem 1. As a consequence, we will deduce our main result (Corollary 18) that the spectrum polynomial $\operatorname{Spec}_{M}(t, q)$ is essentially the generating function for the spectra of $\Delta_{i}^{M}, i=-1, \ldots, r(M)-1$.

Our primary tool will be the exterior face ring $\wedge M$ of $I N(M)$ [2]. Let $W$ be an $\mathbb{R}$-vector space with basis $\left\{v_{e}\right\}_{e \in E}$ indexed by the ground set $E$ of $M$. Let $\wedge W$ be the exterior algebra on $W$, having basis $v_{S}:=v_{e_{1}} \wedge \cdots \wedge v_{e_{s}}$ indexed by subsets $S=\left\{e_{1}, \ldots, e_{s}\right\} \subseteq E$. The exterior face ring $\wedge M$ is the quotient

$$
\wedge M:=\wedge W /\left(v_{S}: S \text { is dependent in } M\right) .
$$

As an $\mathbb{R}$-vector space, we will identify $\wedge M=\bigoplus_{i=0}^{r(M)} \wedge^{i} M$ with the direct sum of the oriented chain groups $\bigoplus_{i=-1}^{r(M)-1} C_{i}^{M}$. As a consequence, this direct sum of chain groups is endowed with an $\mathbb{R}$-algebra structure; under this identification the coboundary maps $\delta_{i}$ correspond to multiplication on the left by the fixed element of degree one $\delta^{M}:=\sum_{e \in E} v_{e}$. Furthermore, for any subset $S \subseteq E$, let $\delta^{S}$ denote the element of degree one $\sum_{s \in S} v_{s}$. The inner product on $\bigoplus_{i} C_{i}^{M}$ corresponds to one on $\wedge M$ in which the $\left\{v_{I}\right\}$ are declared to form an orthonormal basis. With respect to this inner product, the boundary maps $\partial_{i}$ may be thought of as a single map $\partial^{M}$ which is adjoint to left multiplication by $\delta^{M}$, i.e.

$$
\left\langle x, \partial^{M} y\right\rangle=\left\langle\delta^{M} \wedge x, y\right\rangle .
$$

One crucial (and easy to check) property of $\partial^{M}$ is that it is almost a (graded) derivation on the algebra $\wedge M$. We have

$$
\partial^{M}(x \wedge y)=\partial^{M}(x) \wedge y+(-1)^{|x|} x \wedge \partial^{M}(y)
$$

for any $x \in \wedge^{|x|} M$, and $y$ having the property that no $v_{S}, v_{T}$ appearing in $x, y$ respectively have $v_{S} \wedge v_{T}=0$.

We will mostly be dealing with the top-dimensional Laplacian, which for shorter notation we will denote $\Delta^{M}$ rather than $\Delta_{r(M)-1}^{M}$. Similarly let $C^{M}$ replace the notation $C_{r(M)-1}^{M}$ for the top-dimensional chain group. Notice that since there is no chain group in dimension $r(M)$ we have

$$
\Delta^{M}(x)=\delta^{M} \wedge \partial^{M}(x)
$$

for all $x \in \wedge^{r(M)} M$. As a consequence of this fact and our use of $\mathbb{R}$ coefficients, $H^{r(M)-1}(I N(M))$ is a subspace of the 0-eigenspace $\left(\Delta^{M}\right)_{0}$. In fact these two spaces are equal since they have the same dimension.

Note that if $V$ is a flat of $M$, then we can consider $V$ as a matroid by restriction, and $\wedge V$ is naturally a subalgebra of $\wedge M$, so we can think of elements of $\wedge V$ as being in $\wedge M$. Also $\wedge M / V$ is naturally a quotient of $\wedge M$, and by abuse of notation, we can consider elements of $\wedge M / V$ as elements of $\wedge M$ in the following way: if $S \subseteq E-V$ is independent in $M / V$, then identify $v_{S} \in \wedge M / V$ with the element $v_{S} \in \wedge M$. 
Lemma 12. If $x \in \wedge^{r(V)} V$ and $y \in \wedge^{r(M / V)} M / V$ satisfy

$$
\begin{aligned}
\Delta^{V}(x) & =\lambda \cdot x, \\
\Delta^{M / V}(y) & =\mu \cdot y
\end{aligned}
$$

for some real constants $\lambda, \mu$, then

$$
\Delta^{M}(x \wedge y)=(\lambda+\mu) \cdot x \wedge y+\delta^{E-V} \wedge \partial^{M}(x) \wedge y .
$$

Proof. Starting with the left-hand side, we perform a series of manipulations which are justified below:

$$
\begin{aligned}
& \Delta^{M}(x \wedge y) \\
& =\delta^{M} \wedge \partial^{M}(x \wedge y) \\
& =\delta^{M} \wedge \partial^{M}(x) \wedge y+(-1)^{|x|} \delta^{M} \wedge x \wedge \partial^{M} y \\
& =\left(\delta^{V}+\delta^{E-V}\right) \wedge \partial^{M}(x) \wedge y+(-1)^{|x|}\left(\delta^{V}+\delta^{E-V}\right) \wedge x \wedge \partial^{M} y \\
& =\delta^{V} \wedge \partial^{M}(x) \wedge y+\delta^{E-V} \wedge \partial^{M}(x) \wedge y+x \wedge \delta^{V} \wedge \partial^{M} y+x \wedge \delta^{E-V} \wedge \partial^{M} y \\
& =\lambda \cdot x \wedge y+\delta^{E-V} \wedge \partial^{M}(x) \wedge y+0+x \wedge \delta^{E-V} \wedge \partial^{M} y \\
& =\lambda \cdot x \wedge y+\delta^{E-V} \wedge \partial^{M}(x) \wedge y+x \wedge\left(\mu \cdot y+y^{\prime}\right)
\end{aligned}
$$

where $y^{\prime} \in \wedge^{r(M / V)}$ satisfies $x \wedge y^{\prime}=0$ (to be justified below). Assuming this, then the last expression is equal to the right-hand side in the lemma.

Now, the justifications. The first two equalities are by definition of $\Delta^{M}$ and the derivation property of $\partial^{M}$ (equation (4.1)). The third and fourth equalities come from decomposing the sum $\delta^{M}=\delta^{V}+\delta^{E-V}$ and distributing over the wedge product. The fifth equality comes from the facts that

$$
\begin{aligned}
\delta^{V} \wedge \partial^{M}(x) & =\delta^{V} \wedge \partial^{V}(x)(=\lambda \cdot x), \\
x \wedge \delta^{V} & =0
\end{aligned}
$$

which follow since $x$ is supported on bases of $V$. The last equality comes from the fact that

$$
\delta^{M / V} \wedge \partial^{M / V} y=\mu \cdot y \quad \text { in } \wedge M / V
$$

implies

$$
\delta^{E-V} \wedge \partial^{M} y=\mu \cdot y+y^{\prime}
$$

where $y^{\prime}$ is supported on subsets of $E-V$ which are dependent in $M / V$. However all the terms in $y^{\prime}$ will die in $\wedge M$ when one wedges with $x$, since $x$ is supported on a set of bases of $V$.

Corollary 13. With the same conditions and notation of the previous lemma,

(1) if $\lambda=0$, then

$$
\Delta^{M}(x \wedge y)=\mu \cdot x \wedge y
$$

(2) if $r(V)=r(M)-1$ and $y=\delta^{E-V}$, then $\mu=|E-V|$ and

$$
\Delta^{M}(x \wedge y)=(\lambda+|E-V|) \cdot x \wedge y .
$$


Proof. To prove (1), note that $\lambda=0$ implies $x$ is in the 0 -eigenspace of $\Delta^{V}$, which implies that $\partial^{V}(x)=0$. On the other hand, $\partial^{V}(x)=\partial^{M}(x)$ since $x$ is supported on bases of $V$, so the extra term $\delta^{E-V} \wedge \partial^{M}(x) \wedge y$ in the right-hand side of the lemma dies.

To prove (2), the fact that $\Delta^{M / V}(y)=|E-V| y$ when $r(V)=r(M)-1$ and $y=\delta^{E-V}$ is easy to check. Also note that the extra term $\delta^{E-V} \wedge \partial^{M}(x) \wedge y$ dies because $\delta^{E-V} \wedge \delta^{E-V}=0$.

Given a flat $V$ of $M$, we next define a map $h_{V}$,

$$
\begin{aligned}
& h_{V}:\left(\Delta^{V}\right)_{0} \otimes\left(\Delta^{M / V}\right)_{|E-V|} \rightarrow C^{M} \\
& x \otimes y \quad \mapsto x \wedge y,
\end{aligned}
$$

where we recall that $\left(\Delta^{M}\right)_{\lambda}$ denotes the $\lambda$-eigenspace for the operator $\Delta^{M}$. If we let $E(V)$ denote the image of $h_{V}$ in $C^{M}$, then item (1) in Corollary 13 shows that $E(V)$ lies in the $|E-V|$-eigenspace for $\Delta^{M}$. Since $\Delta^{M}$ is a self-adjoint operator, this implies that $E(V), E\left(V^{\prime}\right)$ are orthogonal whenever $V, V^{\prime}$ are flats of different cardinalities. Our goal will be to show more generally that $E(V), E\left(V^{\prime}\right)$ are always orthogonal, and that they give an orthogonal decomposition of the chain group $C^{M}$.

To achieve this goal, and for later purposes, it will be useful to know generators for the eigenspace $\left(\Delta^{M}\right)_{|E|}$. Given any maximal flag

$$
F: \hat{0} \lessdot V_{1} \lessdot \cdots \lessdot V_{r(M)-1} \lessdot M
$$

in the geometric lattice $L(M)$, define $y_{F} \in \wedge M$ by

$$
y_{F}:=\left(\sum_{e \in V_{1}} v_{e}\right) \wedge\left(\sum_{e \in V_{2}-V_{1}} v_{e}\right) \wedge \cdots \wedge\left(\sum_{e \in E-V_{r(M)-1}} v_{e}\right) .
$$

Using induction with item (2) in Corollary 13 shows that $y_{F}$ lies in the eigenspace $\left(\Delta^{M}\right)_{|E|}$. We will not only show that these elements $y_{F}$ span $\left(\Delta^{M}\right)_{|E|}$, but we will also relate them to the top cohomology group $\tilde{H}^{r(M)-2}(L(M))$ of the geometric lattice $L(M)$ (here we are abusing notation by using $L(M)$ to denote both the geometric lattice and the order complex of chains in the proper part of this lattice). Let $C^{r(M)-2}(L(M))$ denote the top cochain group over $\mathbb{R}$ for the order complex of the proper part of $L(M)$, and given a flag $F$ in $L(M)$, let $F^{*}$ denote the cochain which assigns the value 1 to $F$ and 0 to all other flags.

Theorem 14. The map $p: C^{r(M)-2}(L(M)) \rightarrow\left(\Delta^{M}\right)_{|E|}$ sending $F^{*} \mapsto y_{F}$ induces an isomorphism $p_{*}: \tilde{H}^{r(M)-2}(L(M)) \rightarrow\left(\Delta^{M}\right)_{|E|}$.

Proof. First we must verify that $p$ defined as above on the cochains actually gives a well-defined map on cohomology, i.e. $p$ takes $(r(M)-1)$-coboundaries to 0 . Since the $(r(M)-1)$-coboundaries are spanned by the coboundaries of $F^{*}$ where $F$ runs over all flags in $L(M)$ that miss only a single rank, it suffices to show that $p\left(\delta\left(F^{*}\right)\right)=0$ for all such flags $F$. If $F$ is such a flag, and it misses rank $i$, let $V_{i-1}, V_{i+1}$ be its $i-1, i+1$-dimensional flats, respectively. One can quickly check that the computation $p\left(\delta\left(F^{*}\right)\right)=0$ then amounts to a computation in the rank 2 geometric lattice $L\left(V_{i+1} / V_{i-1}\right)$, where it can be easily verified to always work.

Since we have seen before that the dimensions of $\tilde{H}^{r(M)-2}(L(M)),\left(\Delta^{M}\right)_{|E|}$ are both equal to $\left|\mu_{L(M)}(\hat{0}, \hat{1})\right|$, it only remains to show that $p_{*}$ is injective. To do 
this we will show that a particular basis for $\tilde{H}^{r(M)-2}(L(M))$ is mapped to a set of linearly independent elements in $\left(\Delta^{M}\right)|E|$.

Given a base $B$ of $M$ having external activity 0 , let $b_{1}<\cdots<b_{r}$ be the elements of $B$ in their $\omega$-ordering, and let $F(B)$ be the complete flag

$$
\overline{\left\{b_{r}\right\}} \lessdot \overline{\left\{b_{r}, b_{r-1}\right\}} \lessdot \cdots \lessdot \overline{\left\{b_{r}, b_{r-1}, \ldots, b_{2}, b_{1}\right\}} .
$$

In $[1, \S 7.6]$ it is shown that these flags are exactly the ones having decreasing label sets with respect to a certain EL-labelling of the Hasse diagram of $L(M)$. Combining this with $[1,7.6 .3,7.6 .4,7.7 .2]$ and the fact that the Kronecker pairing gives an isomorphism $\tilde{H}_{r(M)-2}(L(M)) \cong \tilde{H}^{r(M)-2}(L(M))$, the cocycles $\left\{F(B)^{*}\right\}$ as $B$ runs over all bases of $M$ of external activity 0 give a basis for $\tilde{H}^{r(M)-2}(L(M))$.

Since $p$ sends $F(B)^{*}$ to $y_{F(B)}$, it would suffice to show the following (cf. [1, proof of Theorem 7.8.4]): for any two bases $B, B^{\prime}$ of external activity 0 , the coefficient of $B$ in $y_{F(B)}$ is \pm 1 , while the coefficient of $B^{\prime}$ is 0 . In proving this, we will use the following two observations, whose straightforward verifications are left to the reader:

(a) If $B$ is a base of external activity 0 , then any non-empty subset $B_{0} \subseteq B$ is a base of external activity zero for the flat $\overline{B_{0}}$ (with respect to the ordering induced from $\omega$ ).

(b) If $B=\left\{b_{1}, \ldots, b_{r}\right\}$ is a base of external activity 0 , as above, and we set $B_{i}=\left\{b_{r}, b_{r-1}, \ldots, b_{i+1}, b_{i}\right\}$, then $b_{i}$ is the $\omega$-smallest element of $\overline{B_{i}}-\overline{B_{i+1}}$.

A typical term in the expansion of $y_{F(B)}$ corresponds to a sequence $\left(x_{r}, \ldots, x_{1}\right)$ with $x_{j} \in \overline{B_{j}}-\overline{B_{j+1}}$ for $1 \leq j \leq r$. We wish to show that $\left\{x_{r}, \ldots, x_{1}\right\}$ is a base of external activity 0 if and only if $x_{j}=b_{j}$ for all $j$. Using induction on $j$ and fact (a) above, it suffices to show that given any $x \in \overline{B_{j}}-\overline{B_{j+1}}$, the set $X=\left\{b_{r}, b_{r-1}, \ldots, b_{j+1}, x\right\}$ is not a base of external activity 0 for $\overline{B_{j}}$. But this follows from fact (b) since $x \in c i_{\overline{B_{j}}}\left(X, b_{j}\right)$.

Remark 15. Combining the isomorphism

$$
p: \tilde{H}^{r(M)-2}(L(M)) \rightarrow\left(\Delta^{M}\right)|E|
$$

from Theorem 14 with the isomorphism

$$
\left(\Delta^{M}\right)_{|E|} \cong\left(\Delta^{M^{*}}\right)_{0}
$$

provided by Proposition 6, we conclude that there is a natural isomorphism

$$
\tilde{H}^{r(M)-2}(L(M)) \cong \tilde{H}_{r\left(M^{*}\right)-1}\left(I N\left(M^{*}\right)\right) .
$$

A. Björner has suggested a different way to see this isomorphism. Let $I N\left(M^{*}\right)^{\vee}$ denote the canonical Alexander dual to $I N\left(M^{*}\right)$, that is,

$$
I N\left(M^{*}\right)^{\vee}:=\left\{F \subseteq E: E-F \notin I N\left(M^{*}\right)\right\}
$$

(see [1, Exercise 7.8.5]). It follows from Alexander duality that there is a natural isomorphism

$$
\tilde{H}_{r\left(M^{*}\right)-1}\left(I N\left(M^{*}\right)\right) \cong \tilde{H}^{r(M)-2}\left(I N\left(M^{*}\right)^{\vee}\right)
$$

On the other hand, one can check using matroid duality that $I N\left(M^{*}\right)^{\vee}$ is exactly the complex of non-spanning subsets of $E$ for $M$, which can be rephrased as saying it 
is the cross-cut complex for the set of atoms in $L(M)$. Hence $I N\left(M^{*}\right)^{\vee}$ is homotopy equivalent to the proper part of $L(M)$, and we have

$$
\tilde{H}^{r(M)-2}\left(I N\left(M^{*}\right)^{\vee}\right) \cong \tilde{H}^{r(M)-2}(L(M)) .
$$

We can now prove one of our main results. Recall that $E(V)$ is defined to be the image of $h_{V}$.

Theorem 16. The map $h:=\bigoplus_{V} h_{V}$,

$$
\bigoplus_{\text {flats } V}\left(\Delta^{V}\right)_{0} \otimes\left(\Delta^{M / V}\right)_{|E-V|} \quad \rightarrow \quad C^{M},
$$

is an isomorphism. Moreover

$$
\bigoplus_{\text {flats } V \text { of } M} E(V)=C^{M}
$$

is an orthogonal direct sum decomposition.

Proof. By Theorem 1, the dimensions of the two spaces are the same, and therefore it suffices to show that $h$ is injective. For this we will show that

(a) for every flat $V$, the map

$$
h_{V}:\left(\Delta^{V}\right)_{0} \otimes\left(\Delta^{M / V}\right)_{|E-V|} \rightarrow C^{M}
$$

is injective, and

(b) for any two different flats $V, V^{\prime}$, the subspaces $E(V), E\left(V^{\prime}\right)$ are orthogonal.

Assertion (a) follows from the fact that $h_{V}$ is a restriction of the injective map

$$
\wedge^{|V|} V \otimes \wedge^{|E-V|} M / V \rightarrow \wedge^{|E|} M
$$

given by $x \otimes y \mapsto x \wedge y$. To see that the latter map is injective, note that it maps a set of basis vectors for the domain into a subset of a basis for the range (the latter subset consisting of all $v_{B}$ indexed by bases $B$ of $M$ which contain a base of $V$ ).

Assertion (b) is a bit trickier, and uses the Laplacian $\Delta^{M}$ in a fundamental way. Let $V, V^{\prime}$ be two different flats of $M$, and let $z=x \wedge y, z^{\prime}=x^{\prime} \wedge y^{\prime}$ be elements of $E(V), E\left(V^{\prime}\right)$, respectively (so $x, x^{\prime}$ are in the eigenspaces $\left(\Delta^{V}\right)_{0},\left(\Delta^{V^{\prime}}\right)_{0}$, respectively, and $y, y^{\prime}$ are in the eigenspaces $\left(\Delta^{M / V}\right)_{|E-V|},\left(\Delta^{M / V^{\prime}}\right)_{\left|E-V^{\prime}\right|}$, respectively). We wish to show $\left\langle z, z^{\prime}\right\rangle=0$.

If $V, V^{\prime}$ are flats of different cardinality, then we are done since then $z, z^{\prime}$ lie in different eigenspaces of the self-adjoint operator $\Delta^{M}$.

If $|V|=\left|V^{\prime}\right|$, then without loss of generality, by Theorem 14 we may assume that $y, y^{\prime}$ are of the form $y_{F}, y_{F^{\prime}}$ for some complete flags $F, F^{\prime}$ in $L(M / V), L\left(M / V^{\prime}\right)$, respectively. Define a new matroid $\tilde{M}$ to be the principal extension of $M$ along the flat $V$ by a new element $p$; in the notation of [3], $\tilde{M}:=M+_{V} p$. We recall a crucial property of principal extensions: any base $B$ of $M$ still forms a base in $\tilde{M}$. In this new matroid $\tilde{M}$, we have two flats $\tilde{V}:=V \cup\{p\}$ and $\tilde{V}^{\prime}=V^{\prime}$ which have different cardinalities, and hence have $E(\tilde{V})$ orthogonal to $E\left(\tilde{V}^{\prime}\right)$ because they lie in different eigenspaces for $\Delta^{\tilde{M}}$. We can still think of $x, x^{\prime}$ as elements of $\wedge \tilde{M}$, and it is easy to see that they will still lie in the eigenspaces $\left(\Delta^{\tilde{V}}\right)_{0},\left(\Delta^{\tilde{V}^{\prime}}\right)_{0}$ because they are killed by $\partial^{\tilde{M}}$. Furthermore, when we think of $y_{F}$ as an element of $\wedge^{\tilde{M} / \tilde{V}}$, it is still in the eigenspace $\left(\Delta^{\tilde{M} / \tilde{V}}\right)_{|E-V|}$ because $\tilde{M} / \tilde{V}=M / V$. On the other hand, if we consider the chain of flats $F^{\prime}$ as a chain $\tilde{F}^{\prime}$ of flats in $L(\tilde{M})$, it generates a 
new element $y_{\tilde{F}^{\prime}}$. One can check that $y_{\tilde{F}^{\prime}}$ differs from $y_{F^{\prime}}$ only in some bases that contain $p$, and hence we have

$$
\begin{aligned}
\left\langle z, z^{\prime}\right\rangle_{M} & =\left\langle x \wedge y_{F}, x^{\prime} \wedge y_{F^{\prime}}\right\rangle_{M} \\
& =\left\langle x \wedge y_{F}, x^{\prime} \wedge y_{\tilde{F}^{\prime}}\right\rangle_{\tilde{M}} \\
& =0 .
\end{aligned}
$$

The only tricky equality here is the second, which relies on the fact that $x \wedge y_{F}$ will not be supported on any bases that contain $p$.

Question 17. Is there any natural way to write down orthogonal projectors

$$
\pi_{V}: C^{M} \rightarrow E(V)
$$

for each flat $V$ of a matroid $M$ ?

In answering this question, we would consider choosing some arbitrary orthonormal basis for each $E(V)$ as not "natural" enough.

We can now state the main corollary to Theorem 16, interpreting the spectra of the Laplacians $\Delta_{i}^{M}$. Recall that $\left(\Delta_{i}^{M}\right)_{\lambda}$ denotes the $\lambda$-eigenspace of $\Delta_{i}^{M}$.

Corollary 18. All Laplacians $\Delta_{i}^{M}$ for any matroid $M$ have integral spectra. Furthermore, the generating function for these spectra is given explicitly by

$$
\sum_{\lambda \in \mathbb{R}, i \geq-1} \operatorname{dim}_{\mathbb{R}}\left(\Delta_{i}^{M}\right)_{\lambda} t^{i} q^{\lambda}=t^{-1} q^{|E|} \operatorname{Spec}_{M}\left(t, q^{-1}\right) .
$$

Proof. Beginning with the left-hand side of the corollary, we perform a sequence of manipulations which are justified below:

$$
\begin{aligned}
& \sum_{\lambda \in \mathbb{R}, i \geq-1} \operatorname{dim}_{\mathbb{R}}\left(\Delta_{i}^{M}\right)_{\lambda} t^{i} q^{\lambda} \\
= & \sum_{\text {flats } V \text { of } M} t^{r(V)-1} \sum_{\lambda \in \mathbb{R}} \operatorname{dim}_{\mathbb{R}}\left(\Delta_{r(V)-1}^{V}\right)_{\lambda} q^{\lambda+|E-V|} \\
= & \sum_{\text {flats } V \text { of } M} t^{r(V)-1} \sum_{\text {flats } V^{\prime} \subseteq V} \operatorname{dim}_{\mathbb{R}}\left(\Delta_{r\left(V^{\prime}\right)-1}^{V^{\prime}}\right)_{0} \\
= & \sum_{\text {flats } V \text { of } M} t^{r(V)-1} \sum_{\text {flats } V^{\prime} \subseteq V}\left|\tilde{\chi}\left(I N\left(V^{\prime}\right)\right)\right| \cdot\left|\mu_{L(M)}\left(V^{\prime}, V\right)\right| q^{\left|E-V^{\prime}\right|} \\
= & t^{-1} q^{|E|} \operatorname{Spec}_{M}\left(t, q^{-1}\right) .
\end{aligned}
$$

The first equality above is justified by Proposition 5 , the second equality by Theorem 16 and Corollary 13, the third equality by the same facts from [1] that were used in the proof of Theorem 1, and the last equality by equation (2.2).

\section{SymmetriC EXAMPLES}

In [8], the analysis of the eigenspaces of the Laplacians for the chessboard complexes necessarily also entailed a description of these eigenspaces as representations for the symmetry groups present. Similarly, we can ask for such a description for the eigenspaces of the Laplacians of a matroid complex whenever there are nontrivial automorphisms of the matroid. This section is devoted to some examples of this nature. 
We begin with a reduction. Combining Theorem 16 and Proposition 5 gives the isomorphism

$$
\begin{aligned}
C_{i}^{M} & \cong \bigoplus_{\text {flats } V \text { with } r(V)=i+1} \bigoplus_{\text {flats } V^{\prime} \subseteq V}\left(\Delta_{r\left(V^{\prime}\right)-1}^{V^{\prime}}\right)_{0} \otimes\left(\Delta_{r\left(V / V^{\prime}\right)-1}^{V / V^{\prime}}\right)_{\left|V-V^{\prime}\right|} \\
& \cong \bigoplus_{\text {flats } V \text { with } r(V)=i+1} \bigoplus_{\text {flats } V^{\prime} \subseteq V} \tilde{H}_{r\left(V^{\prime}\right)-1}\left(I N\left(V^{\prime}\right)\right) \otimes \tilde{H}^{r\left(V / V^{\prime}\right)-2}\left(L\left(V / V^{\prime}\right)\right) .
\end{aligned}
$$

Let $G$ be a group of automorphisms of the matroid $M$. Note that $G$ permutes the chains of flats $V^{\prime} \subseteq V$, and the stabilizer

$$
\operatorname{Stab}_{G}\left(V^{\prime}, V\right):=\left\{g \in G: g V^{\prime} \subseteq V^{\prime}, g V \subseteq V\right\}
$$

acts on $\tilde{H}_{r\left(V^{\prime}\right)-1}\left(I N\left(V^{\prime}\right)\right)$ and $\tilde{H}^{r\left(V / V^{\prime}\right)-2}\left(L\left(V / V^{\prime}\right)\right)$. Since the map $h$ in Theorem 16 is easily checked to be $G$-equivariant, equation (5.1) leads immediately to the following result:

Theorem 19. Let $G$ be a group of automorphisms of $M$. Then as $G$-representations we have isomorphisms

$$
C_{i}^{M} \cong \bigoplus_{\left(V^{\prime}, V\right)} \operatorname{Ind}_{S t a b_{G}\left(V^{\prime}, V\right)}^{G} \tilde{H}_{r\left(V^{\prime}\right)-1}\left(I N\left(V^{\prime}\right)\right) \otimes \tilde{H}^{r\left(V / V^{\prime}\right)-2}\left(L\left(V / V^{\prime}\right)\right)
$$

as $\left(V^{\prime}, V\right)$ runs over a set of representatives of all $G$-orbits of chains of flats $V^{\prime} \subseteq V$ having $r(V)=i+1$.

In the statement of Theorem 19, $\operatorname{Ind}_{S t a b_{G}\left(V^{\prime}, V\right)}^{G} W$ denotes the induction of a representation $W$ of the subgroup $\operatorname{Stab}_{G}\left(V^{\prime}, V\right)$ to the group $G$.

Note that all flats $\left(V^{\prime}, V\right)$ in a single $G$-orbit will have $V^{\prime}$ of the same cardinality, and therefore their corresponding spaces $\tilde{H}_{r\left(V^{\prime}\right)-1}\left(I N\left(V^{\prime}\right)\right) \otimes \tilde{H}^{r\left(V / V^{\prime}\right)-2}\left(L\left(V / V^{\prime}\right)\right)$ will lie in the same eigenspace of $\Delta_{i}^{M}$. This implies that the decomposition of $G$ representations in Theorem 19 is still finer than the decomposition into eigenspaces of $\Delta_{i}^{M}$.

The previous result suggests that we concentrate attention on understanding the $G$-action on the homology of the matroid complex $I N(M)$. The actions on the cohomology of the geometric lattice $L(M)$ tend to have been studied earlier, or we can always use the isomorphism

$$
\tilde{H}^{r(M)-2}(L(M)) \cong\left(\Delta^{M}\right)_{|E|} \cong\left(\Delta^{M^{*}}\right)_{0} \cong \tilde{H}_{r\left(M^{*}\right)-1}\left(I N\left(M^{*}\right)\right)
$$

to reduce to the study of $I N\left(M^{*}\right)$ when this is convenient.

Notice that in general one can write down the $G$-action on $H_{r(M)-1}(I N(M))$ as a virtual character using the Euler characteristic:

$$
\tilde{H}_{r(M)-1}(I N(M)) \cong \sum_{i \geq-1}(-1)^{r(M)-i-1} C_{i}^{M} .
$$

However it is not always clear why this expression gives a genuine character rather than just a virtual one.

Example 1. Uniform matroids $U(r, n)$. The uniform matroid $M=U(r, n)$ of rank $r$ on ground set $[n]:=\{1,2, \ldots, n\}$ has as independent sets all subsets of cardinality at most $r$. Flats, contractions, and duals of uniform matroids are all again uniform. 
The symmetric group $G=S_{n}$ acts on $U(r, n)$ by permuting the ground set $[n]$, and the $G$-orbits of chains of flats $V^{\prime} \subseteq V$ are determined by the cardinality of the sets $V^{\prime}, V$. In light of this discussion, it suffices to determine the $S_{n}$-action on $I N(U(r, n))$. We can use equation (5.4), once we determine the $S_{n}$-action on $C_{i}^{M}$. The space $C_{i}^{M}$ is the span of all oriented $i$-simplices $\left[j_{0}, \ldots, j_{i}\right]$. These $i$-simplices all lie in a single $S_{n}$-orbit. The stabilizer of a fixed oriented $i$-simplex is a subgroup isomorphic to $S_{i+1} \times S_{n-i-1}$, and we observe that the stabilizer acts on the 1dimensional space spanned by this fixed oriented $i$-simplex by the sign character sgn of $S_{i+1}$ tensored with the trivial character 1 of $S_{n-i-1}$. We conclude that

$$
C_{i}^{M} \cong \operatorname{Ind}_{S_{i+1} \times S_{n-i-1}}^{S_{n}} \operatorname{sgn} \otimes 1 .
$$

Using the Littlewood-Richardson rule $[10, \S 4.9]$ along with equation (5.4) then shows that $I N(U(r, n))$ carries the irreducible $S_{n}$-representation indexed by the partition shape $\left(n-r, 1^{r}\right)$ (see [10, Chapter 2]).

Example 2. Finite projective spaces $\mathbb{P}_{\mathbb{F}_{q}}^{n-1}$. Let $\mathbb{F}_{q}$ be the finite field with $q$ elements, for $q$ a prime power, and $\mathbb{F}_{q}^{n}$ an $n$-dimensional space over $\mathbb{F}_{q}$. The projectivization of this space forms a matroid $M=\mathbb{P}_{\mathbb{F}_{q}}^{n-1}$ whose ground set is the set of lines through the origin in $\mathbb{F}_{q}^{n}$, and where a subset of lines is independent if and only if they contain a set of representing vectors which are linearly independent in the usual sense. Flats and contractions of finite projective spaces are again isomorphic to finite projective spaces. One might suspect from the terminology that dual matroids of finite projective spaces are again isomorphic as matroids to finite projective spaces (for the dual space), but this is not true as shown by simple examples.

The finite general linear group $G L_{n}\left(\mathbb{F}_{q}\right)$ acts on $\mathbb{P}_{\mathbb{F}_{q}}^{n-1}$ in the obvious way and the $G$-orbits of chains of flats $V^{\prime} \subseteq V$ are determined by the dimension of $V^{\prime}, V$ as subspaces. In light of this discussion, it suffices to determine the $G L_{n}\left(\mathbb{F}_{q}\right)$-action on $\tilde{H}^{n-1}\left(L\left(\mathbb{P}_{\mathbb{F}_{q}}^{n-1}\right)\right)$ and on $\tilde{H}_{n-1}\left(I N\left(\mathbb{P}_{\mathbb{F}_{q}}^{n-1}\right)\right)$. The first task is relatively easy since $L\left(\mathbb{P}_{\mathbb{F}_{q}}^{n-1}\right)$ is the finite vector space lattice, whose order complex is the Tits building for the usual $B N$-pair structure on $G L_{n}\left(\mathbb{F}_{q}\right)$. Here the $G L_{n}\left(\mathbb{F}_{q}\right)$-representation on the top (co-)homology group $\tilde{H}^{n-1}\left(L\left(\mathbb{P}_{\mathbb{F}_{q}}^{n-1}\right)\right)$ is the well-known Steinberg character [11], an irreducible representation of $G L_{n}\left(\mathbb{F}_{q}\right)$ of dimension $q^{\left(\begin{array}{c}n \\ 2\end{array}\right)}$.

To describe the $G L_{n}\left(\mathbb{F}_{q}\right)$-representation on $I N\left(\mathbb{P}_{\mathbb{F}_{q}}^{n-1}\right)$, we can try to be more explicit in equation (5.4), by describing the $G L_{n}\left(\mathbb{F}_{q}\right)$-action on $C_{i}^{M}$. The space $C_{i}^{M}$ is the span of all oriented $i$-simplices $\left[l_{0}, \ldots, l_{i}\right]$, for sets $\left\{l_{0}, \ldots, l_{i}\right\}$ of independent lines through the origin. Such $i$-simplicies all lie in a single $G L_{n}\left(\mathbb{F}_{q}\right)$-orbit, and the stabilizer of a fixed oriented $i$-simplex is a subgroup $H$ isomorphic to the following subgroup of $2 \times 2$ block upper-triangular matrices:

$$
\left(\begin{array}{ll}
A & B \\
0 & C
\end{array}\right)
$$

Here $A$ must be an $(i+1) \times(i+1)$ monomial matrix, i.e. it has exactly one nonzero entry in each row and column, $B$ is an arbitrary $(i+1) \times(n-i-1)$ matrix, and $C$ is an invertible $(n-i-1) \times(n-i-1)$ matrix. Elements of the stabilizer $H$ act on the oriented simplex which they fix by the sign of the permutation associated to the monomial matrix $A$, so call this character $\chi$. We conclude that $C_{i}^{M} \cong \operatorname{Ind}_{H}^{G L_{n}\left(\mathbb{F}_{q}\right)} \chi$, and equation (5.4) gives an expression for $\tilde{H}_{n-1}\left(I N\left(\mathbb{P}_{\mathbb{F}_{q}}^{n-1}\right)\right)$ as a virtual character 
of dimension

$$
\sum_{i \geq-1}(-1)^{n-1-i} \frac{[n]_{q}[n-1]_{q} \cdots[n-i]_{q}}{(i+1) !} q^{\left(\begin{array}{c}
i+1 \\
2
\end{array}\right)}
$$

where $[n]_{q}:=1+q+q^{2}+\cdots+q^{n-1}$. We do not see how to simplify the expression (5.4) for $\tilde{H}_{n-1}\left(I N\left(\mathbb{P}_{\mathbb{F}_{q}}^{n-1}\right)\right)$ as a virtual character.

Example 3. The graphic matroid for a complete graph. Let $M_{n}^{A}$ be the graphic matroid associated to the complete graph on vertex set $[n]$. In other words, $M_{n}^{A}$ has as ground set $E$ the set of all possible edges $\{i, j\}$ with $1 \leq i<j \leq n$, and a set of edges is independent if it contains no cycles.

The symmetric group $S_{n}$ acts on $M_{n}^{A}$ by permuting the vertex set [ $\left.n\right]$, and hence also permuting the edges $\{i, j\}$. Flats in $M_{n}^{A}$ correspond to partitions $\pi=B_{1}\left|B_{2}\right| \cdots \mid B_{r}$ of $[n]$ into blocks $B_{i}$, and one can check that if $V$ is the flat corresponding to the partition $\pi$, then as a matroid $V \cong \bigoplus_{i} M_{\left|B_{i}\right|}^{A}$. The lattice $L\left(M_{n}^{A}\right)$ is the well-known lattice of set partitions of $[n]$, usually denoted $\Pi_{n}$. Furthermore, if $V^{\prime}$ is a flat contained in $V$, then $V^{\prime}$ corresponds to a partition $\pi^{\prime}$ which refines $\pi$, and $L\left(V / V^{\prime}\right)$ is isomorphic to a product of lattices of the form $\Pi_{n_{i}}$ for some $n_{i}<n$.

In light of this discussion, it suffices to determine the $S_{n}$-action on $\tilde{H}^{n-3}\left(\Pi_{n}\right)$ and on $\tilde{H}_{n-2}\left(I N\left(M_{n}^{A}\right)\right)$. The first task is relatively easy since the $S_{n}$-action on $\tilde{H}^{n-3}\left(\Pi_{n}\right)$ has been well-studied by many authors; see e.g. [12, $\S 7$. It is known that as an $S_{n}$-representation,

$$
\tilde{H}^{n-3}\left(\Pi_{n}\right) \cong \operatorname{sgn} \otimes \operatorname{Ind}_{C_{n}}^{S_{n}} \zeta
$$

where $s g n$ is the 1-dimensional sign representation, $C_{n}$ is the cyclic group in $S_{n}$ generated by an $n$-cycle, and $\zeta$ is the 1-dimensional representation of $C_{n}$ which sends a generator to a primitive $n^{t h}$ root of unity [12, Theorem 7.3]. It is also known that if we let $S_{n-1}$ denote the subgroup of $S_{n}$ which fixes $n$, then

$$
\operatorname{Res}_{S_{n-1}}^{S_{n}} \tilde{H}^{n-3}\left(\Pi_{n}\right) \cong \mathbb{R}\left[S_{n-1}\right]
$$

i.e. the restriction to $S_{n-1}$ is isomorphic to the regular representation of $S_{n-1}$ [12, Corollary 7.6]. Consequently, this representation has dimension $(n-1)$ !.

The $S_{n}$-action on $\tilde{H}_{n-2}\left(I N\left(M_{n}^{A}\right)\right)$ has only been studied recently in [9]. The description of the $S_{n}$-representation is not known in general, although equation (5.4) can be used to decompose it into $S_{n}$-irreducibles via a character computation when $n$ is small. On the other hand, one can construct an explicit basis for the homology which is permuted by the action of $S_{n-1}$ and which shows the following:

Theorem 20 ([9, Theorem 3.6.3]). As an $S_{n-1}$-representation $\tilde{H}_{n-2}\left(I N\left(M_{n}^{A}\right)\right)$ is isomorphic to the permutation action on forests of edge-rooted trees on $[n-1]$ tensored with the sign character sgn.

Here a forest of edge-rooted trees is a graph on $[n-1]$ in which every connected component is a tree, along with a specified edge of each tree (so in particular, every vertex in $[n-1]$ lies in a tree having at least two vertices). We give a brief proof of this result here, relying on results of [1].

First, choose the ordering $\omega$ of the edges in the complete graph as follows: an edge $\{i, j\}$ with $i<j$ is earlier in $\omega$ than $\left\{i^{\prime}, j^{\prime}\right\}$ if $j>j^{\prime}$ or if $j=j^{\prime}$ and $i>i^{\prime}$. In 

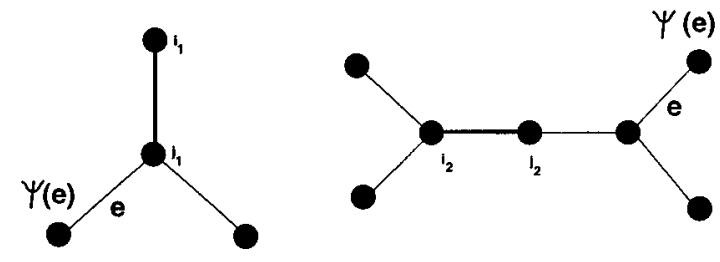

FiguRE 2. Example of an edge rooted-forest along with some nonroot edges $e$ and their corresponding vertices $\psi(e)$.

other words,

$$
\{n-1, n\}<_{\omega} \cdots<_{\omega}\{1, n\}<_{\omega}\{n-2, n-1\}<_{\omega} \cdots<_{\omega}\{2,3\}<_{\omega}\{1,3\}<_{\omega}\{1,2\} .
$$

Bases of $M_{n}^{A}$ correspond to spanning trees $T$ on $[n]$, and it is straightforward to check the following characterization of the bases of internal activity 0 :

Lemma 21. A spanning tree $T$ on $[n]$ has internal activity 0 if and only if for every tree of the forest $\left.T\right|_{[n-1]}$, the vertex connecting this tree to $n$ does not have the maximum label in the component.

Here $\left.T\right|_{[n-1]}$ denotes the restriction of the tree $T$ to its edges which only contain vertices in $[n-1]$.

If we let $T_{1}, \ldots, T_{r}$ denote the trees of internal activity 0 , then $[1$, Theorem 7.7.2] implies that there is a basis of cycles $z_{1}, \ldots, z_{r}$ for $\tilde{H}_{n-2}\left(I N\left(M_{n}^{A}\right)\right)$ uniquely determined as homology classes by the condition that the coefficient of $T_{i}$ in $z_{j}$ is 1 if $i=j$ and 0 else. These cycles $\left\{z_{i}\right\}$ turn out not to be permuted by the $S_{n-1}$-action, however they are upper-triangularly related to a set of cycles which are so permuted, and which we now define.

Given a forest $F$ of edge-rooted trees on $[n-1]$, let $F_{1}, \ldots, F_{s}$ be its component edge-rooted trees, let the distinguished edge in tree $F_{r}$ be $\left\{i_{r}, j_{r}\right\}$, and for any other edge $e \neq\left\{i_{r}, j_{r}\right\}$ in $F_{r}$ let $\psi(e)$ be the vertex in this edge which lies farthest from the root edge. See Figure 2 for some examples.

Define $z_{F}^{\prime}$ to be the following product in the exterior face ring $\wedge M_{n}^{A}$ :

$$
z_{F}^{\prime}:=\bigwedge_{r=1}^{s}\left(\partial\left(v_{i_{r} j_{r}} \wedge v_{i_{r} n} \wedge v_{j_{r} n}\right) \wedge \bigwedge_{\text {edges } e \neq\left\{i_{r}, j_{r}\right\} \text { in } F_{r}} \partial\left(v_{e} \wedge v_{\psi(e), n}\right)\right)
$$

We next describe a bijection $\phi$ between the forests of edge-rooted trees on $[n-1]$ and the trees of internal activity 0 on $[n]$. Given such a forest $F$ let $\phi(F)$ be the tree on $[n]$ obtained as follows: for each edge-rooted tree $F_{r}$ of $F$, assume that the labelling $i_{r}, j_{r}$ of the distinguished edge has been chosen so that $i_{r}$ is farther than $j_{r}$ is from the maximum labelled vertex of $F_{r}$. Then $\phi(F)$ is obtained by adding all the edges $\left\{i_{r}, n\right\}$ for $1 \leq r \leq s$ to $F$. It is easy to see that $\phi(F)$ fits the description of Lemma 21 , and so has internal activity 0 . The inverse map $\phi^{-1}$ starts with a tree $T$ of internal activity 0 , removes all edges involving the vertex $n$ leaving a forest on $[n-1]$, and for each vertex $i$ that used to be connected to $n$ it distinguishes the edge containing $i$ in the forest which is the first edge on the path toward the maximum labelled vertex of $i$ 's component tree. 
We now come to the crucial point. Given two forests $F_{i}, F_{j}$ of edge-rooted trees, having corresponding trees $T_{i}, T_{j}$ of internal activity zero, if the cycle $z_{F_{i}}^{\prime}$ contains the tree $T_{j}$, then one can easily check that:

(a) as a forest, $F_{i}$ contains all the edges in $F_{j}$, and furthermore

(b) if $F_{i}$ and $F_{j}$ have exactly the same set of edges as forests, then in each of their component trees, the distinguished edge for $F_{j}$ lies on the shortest path connecting the distinguished edge for $F_{i}$ to the maximum labelled vertex in that component.

Consequently, if we linearly order the forests of edge-rooted trees by any linear extension of the partial order expressed in items (a), (b) above, we have the following property: when one restricts the matrix expressing the cycles $z_{F_{i}}^{\prime}$ 's in terms of trees on $[n]$ to the trees of internal activity 0 , the result is a square upper-triangular matrix $U$ with \pm 1 's on the diagonal. By [1, Theorem 7.7.2], this implies the cycles $\left\{U^{-1} z_{F}^{\prime}\right\}$ must induce the exact same homology classes as the basis $\left\{z_{i}\right\}$ mentioned earlier for $\tilde{H}_{n-2}\left(I N\left(M_{n}^{A}\right)\right)$. The next corollary is then immediate:

Corollary 22. The cycles $\left\{z_{F}^{\prime}\right\}$ as $F$ ranges over all forests of edge-rooted trees form a basis for $\tilde{H}_{n-2}\left(I N\left(M_{n}^{A}\right)\right)$.

It is easy to see that permutations in $S_{n-1}$ permute the cycles $\left\{z_{F}^{\prime}\right\}$ (up to sign) in the same way that they permute the forests of edge-rooted trees. Furthermore, it is not hard to check that if $F$ is a forest of edge-rooted trees which is fixed by some permutation $\pi$ in $S_{n-1}$, then we also have $\pi\left(z_{F}^{\prime}\right)=\operatorname{sgn}(\pi) \cdot z_{F}^{\prime}$. This proves Theorem 20.

\section{ACKNOWLEDGMENTS}

The authors would like to thank Paul Edelman for helpful comments.

Note added in proof. Our results have recently been generalized in two directions by G. Denham ("The combinatorial Laplacian of the Tutte complex", preprint 1999, available at www.math.1sa.umich.edu/ denham/research.html). He gives a very similar analysis of the spectrum for a more general Laplace operator, involving weights for each element of the ground set, and which acts on a chain complex larger than the simplicial chains of $I N(M)$. In so doing, he produces a very general invariant of the matroid $M$, which can be specialized either to our spectrum polynomial $\operatorname{Spec}_{M}(t, q)$ or to the Tutte polynomial $T_{M}(x, y)$.

\section{REFERENCES}

[1] A. Björner, The homology and shellability of matroids and geometric lattices, in Matroid Applications (ed. N. White), Encyclopedia of Mathematics and Its Applications, 40, Cambridge Univ. Press, 1992. MR 94a:52030

[2] A. Björner and G. Kalai, On f-vectors and homology, in Combinatorial Mathematics: Proc. of 3rd International Conference (New York, 1985), N.Y. Acad. Sci.: New York, 1989. MR 90i: 52008

[3] T. Brylawski, Constructions, in Theory of Matroids (ed. N. White), Encyclopedia of Mathematics and Its Applications, 26, Cambridge Univ. Press, 1986. CMP 18:15

[4] T. Brylawski and J. G. Oxley, The Tutte polynomial and its applications, in Matroid Applications (ed. N. White), Encyclopedia of Mathematics and Its Applications, 40, Cambridge Univ. Press, 1992. MR 93k:05060

[5] G. Etienne and M. Las Vergnas, External and internal elements of a matroid basis, Disc. Math. 179 (1998), 111-119. MR 98m:05038 
[6] J. Friedman, Computing Betti numbers via combinatorial Laplacians, in Proc. 28th Annual ACM Symposium on the Theory of Computing, ACM: New York, 1996, 386-391. MR 97g:68005

[7] J. Friedman, Computing Betti numbers via combinatorial Laplacians, Algorithmica 21 (1998), 331-346. MR 99c:52022

[8] J. Friedman and P. Hanlon, On the Betti numbers of chessboard complexes, J. Algebraic Combin. 8 (1998), 193-203. CMP 99:02

[9] W. Kook, Categories of acyclic graphs and automorphisms of free groups, Ph.D. thesis (G. Carlsson, advisor), Stanford Univ., 1996.

[10] B. Sagan, The symmetric group: representations, combinatorial algorithms, and symmetric functions, Wadsworth \& Brooks/Cole: Pacific Grove, 1991. MR 93f:05102

[11] L. Solomon, The Steinberg character of a finite group with a BN-pair, in Theory of Finite Groups (eds. R. Brauer and C. H. Sah), Benjamin: New York, 1969, 213-221. MR 40:220

[12] R. Stanley, Some aspects of groups acting on finite posets, J. Combin. Theory Ser. A 32 (1982), 132-161. MR 83d:06002

School of Mathematics, University of Minnesota, Minneapolis, Minnesota 55455

E-mail address: kook@math.umn.edu

Current address, W. Kook: Department of Mathematics, The George Washington University, Washington DC 20052

E-mail address: andrewk@gwu.edu

E-mail address: reiner@math.umn.edu

E-mail address: stanton@math.umn.edu 